Aziaka D. S.

The Influence of Rescheduled-Overhaul in determining the number of Gas turbine Usage in Natural Gas Pipeline Transportation Network

\author{
d.aziaka@cranfield.ac.uk \\ Centre for Power and Propulsion, \\ Cranfield University, \\ Cranfield Bedfordshire. UK \\ This article is covered and protected by copyright law and all rights reserved exclusively by the \\ Centre for Petroleum, Pollution Control and Corrosion Studies. \\ (CEFPACS) Consulting Limited. \\ Electronic copies available to authorised users.
}

The link to this publication is https://ajoeer.org.ng/otn/ajoeer/qtr-1/2020/01 


\title{
The Influence of Rescheduled-Overhaul in determining the number of Gas turbine Usage in Natural Gas Pipeline Transportation Network
}

\author{
Aziaka D. $\mathbf{S}^{1^{*}}$; Le-ol A. $\mathrm{K}^{2}$; Pilidis $\mathbf{P}^{3}$. \\ ${ }^{1,3}$ Centre for Power and Propulsion, Cranfield University, Cranfield Bedfordshire \\ ${ }^{2}$ Department of Mechanical Engineering, Rivers State University, Port Harcourt, Nigeria \\ d.aziaka@cranfield.ac.uk; le-ol.anthony@ust.edu.ng; p.pilidis@cranfield.ac.uk
}

[Date received: Jan 2020. Date accepted: Feb 2020]

\begin{abstract}
:
Gas turbines are an integral part of the supply of natural gas in many parts of the world. In compressor stations, they are used for transporting gas from producing wells to consumers and across extensive networks of pipelines. This paper present how rescheduling of gas turbine overhaul from the baseline condition amid degradation influences the number of the gas turbine to be used in a compressor station without altering the original pipeline design for the desired amount of gas delivery. Eighteen compressor stations with gas turbine engines as the driver to the gas compressor have been investigated. The selected engines models were developed based on public domain specification, using an in-house engine performance simulation software: TURBOMATCH. Three seasons (rainy, dry and hot seasons) were considered in this paper based on the location of Trans-Saharan gas pipeline being investigated. Compressor and turbine were degraded (fouled) as a single type of degradation producing three performance scenarios (optimistic, medium and pessimistic). These scenarios define the levels of deterioration of the gas turbine in comparison with the clean conditions. The baseline case indicated that at a controlled TET, the number of GT used in each compressor station increases with increase in degradation (reduction in flow capacity and isentropic efficiency) which result to a variation in the number of engines per station. The result revealed that the implementation of rescheduled-overhaul on the engines reduces the number of Gas turbine usage at the same degraded and ambient condition of the baseline case. The further result indicated that the optimistic, medium and pessimistic scenarios that used 99, 106 and 120 number of engines for the 18 compression stations at baseline condition reduces to 91, 104 and 115 respectively when rescheduled overhaul was implemented for the same amount of gas to be delivered and at the same operating conditions. The proposed approach will enhance engine life-extension strategies that engine life-cycle managers, or natural gas pipeline investors may adopt to cost-effectively manage their engines while ensuring reliability and safety on the pipeline business.
\end{abstract}

Keywords: Turbine Entry Temperature, Gas Compressor, TURBOMATCH, Natural gas, Degradation 


\section{NOMENCLATURE}

API---American petroleum institute

CS---Compressor Station

DP---Design point

D.Season--Dry season

EFF---Efficiency

EGT-Exhaust gas temperature

ETASF---Efficiency scaling factor

FF---Fuel flow

GC---Gas compressor

GT---Gas Turbine

H.Season---Hot season

Med-Medium

OEM--Original engine manufacturer

Opt-Optimistic
Pess- Pessimistic

PM-Prime mover

$P R_{\text {Map }}$ - Pressure ratio of the default map

PRSF- Pressure ratio scaling factor

ROH-Rescheduled overhaul

R.Season- Rainy season

TET-Turbine entry temperature

$T F_{D P, M a p}$-Turbine flow for default map

$T F_{D P}$-Turbine flow for a new map

TFSF-Turbine flow scaling factor

TSGP-Trans-Saharan Gas Pipeline

$W A C_{D P}$-Mass flow design point for a new map

$W A C_{D P}$ Map- Default map mass flow design pointt

WASF- Mass flow scaling factor

\subsection{Introduction}

How long the Gas turbine (GT) engines can be kept in service safely during the project life amid harsh ambient temperature and degradation has become a growing concern to engine life-cycle managers. Also, the need to balance risk and cost of replacing completely deteriorated engines explains the growing interest in the application of life extension technologies or strategies for safely extracting maximum usage of the procured engines on the natural gas pipeline. The reason is that the engines are allowed to run for an extended period due to the economic benefits of the natural gas business[1-3]. GT engines that work for an extended period need overhaul to revamp the engine and to keep the engine working to its useful life[4].

It is important to state that, a gas-fired turbine in natural gas transmission service is much smaller than a "typical" unit employed in the power sector. The unit size in gas transmission service makes the engine amenable to an overhauled approach that may not be possible for other application [4].To enhance a reliable and safe service, the gas turbine engine, like all mechanical equipment, need a planned maintenance program that is dependable with the operating environment. Such plans generally consist of frequent inspections, routine 
servicing, and component replacement. Engine overhaul is performed as a result of routine inspections that revealed operational or mechanical deficiencies such as high equipment vibration levels, abnormal oil temperatures, or visible damage and the deterioration of subcomponents. Anyone of these inspection results could trigger routine maintenance activities that range from minor repairs to overhauling [5].

The compressor is vulnerable to dust and particles which generally affect GT operation. Also, compressor turbine and power turbine portions of the gas-fired turbine are exposed to hightemperature combustion gases, along with vibration and thermal cycling, which result to mechanical stresses, thermal stresses, and corrosion in these sections of the turbine. Hence the need to employ engine overhaul periodically to maintain the engine and minimize breakdown.

Beal [4] explained that inspection, disassembly, repair and or replacement of subcomponents, assembly, and test of the gas generator and power turbine component is an integral part of the overhaul process.

NSPS [6] presented that most GT engines are designed for 20,000 to 40,000 operating hours between overhaul. Also, most of the original engine manufacturer (OEM) such as GE, Solar, and Rolls Royce jointly represent about $87 \%$ of GT in the natural gas pipeline industry, and all three manufacturers recommend scheduled GT engine overhauls at approximately 30,000 hours of service [6]. However, the time interval varies with make, model and engine condition of operation which may extend or reduce to longer or shorter service intervals.

Given the above explanation, NSPS [6] finalised that most GT engines in natural gas pipeline transportation network are subject to overhaul at an interval of every three to five years of operation. In some cases, overhaul can take place more often, subject to operational performance and the results of inspections. Based on the above presentation, Aziaka [7] investigated the effect of implementing three years overhaul interval on the techno-economic assessment of the natural gas pipeline network and recommended that period between overhaul should be changed due to the adverse effect of degradation of GT engine at the compression station to investigates its impacts on the system. 
Therefore, this paper presents the influence of rescheduled overhaul from three years interval to two years based on engine degradation in determining the number of GT usage in each compression station. The research is necessary because the time interval varies with engine make, model and conditions of operation.

According to Wulf [8], GT engines returns to operation with some level of degradation after each maintenance cycle. The returned percentage of degradation increases as the operation continues with time. This implies that as the engine continues operation after each segment of the overhaul, the unrecovered degradation increases with additional maintenance cycles [42].

\subsection{Methodology}

The baseline model instituted three years interval for the engine overhaul as investigated by Aziaka [7]. However, Figure 1 represents the developed method for implementing the concept of gas turbine re-scheduled overhaul $(\mathrm{ROH})$ for the natural gas pipeline project from three to two years interval. First of all, the gas compressor(GC) power requirement in each compression station needs to be ascertained before the determination of the capacity of GT to be used. The overhaul was implemented after the two years interval of each season of the year. The engine model with the implementation of the re-scheduled overhaul was developed in TURBOMATCH. This was done amid time-based ambient temperature and the different level of degradation (opt, med and pess) for each season of the year for thirty (30) years. The limit of the operational TET not exceeding 60k above the DP was also considered irrespective of the level of the engine degradation. This concept is discussed below.

Based on the use of the different number of engines with their respective shaft power, conjugate TET performance maps of the various power settings of the engines will be produced. The produced TET map, having satisfied the condition of operation will form the bases of determining the exact number of GT to be used for the project life. 


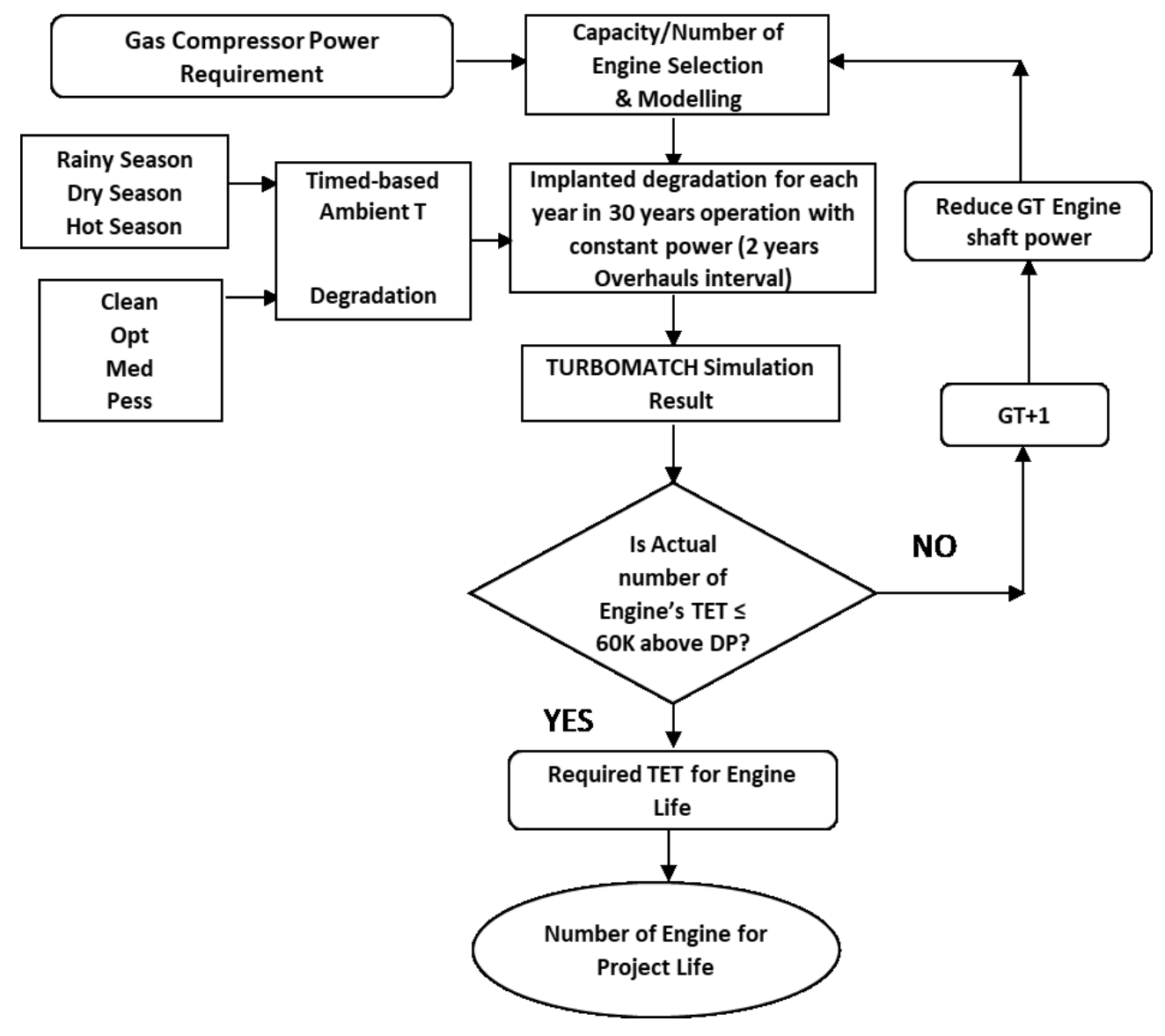

Fig 1: Methodology for the re-scheduled overhaul

\subsection{Performance Simulation and Specification of Case Study Modelled Gas Turbine Engine.}

TURBOMATCH software was used for the modelling and performance simulation, including the degraded (fouling) condition of the selected mixed-fleets engines. TURBOMATCH is an advance in-house software of Cranfield University in the United Kingdom used to model and simulate gas turbine engine at both design and Off-design performance using a modified Newton-Raphson method as the convergence technique. Design point (DP) calculation was carried out with initial user specification of component efficiency, ambient condition, pressure loss, EGT, etc. The off-design (OD) compressor and turbine component operating point on their maps was determined based on their calculated scaling factors as represented in Equation. (1) to (8). The engine was modelled with a fixed shaft power. 
Four GTs that are inspired by SGT-400, SGT-500, GT35 and LM1600, referred to as IGTA, IGTB, IGTC and IGTD respectively as represented in Figure 2 was modelled in TURBOMATCH. The engines were modelled and built based on the public domain data of the inspired engines as given by the OEM. Based on the performance simulation result, the individual gas turbine was compared based on performance data from the original engine manufacturer (OEM) in order to ascertain the accuracy of the model and was found $99 \%$ accurate at DP [9]. It is worthy of note that a $10 \%$ margin on the power of the inspired gas turbine selected for the compressor drive as required by API 616 was considered as the minimum margin power based on the temperature profile of the Trans-Saharan gas pipeline (TSGP). These four engines were distributed to the eighteen compressor stations based on GC power requirement for each station as shown in Table 1.0

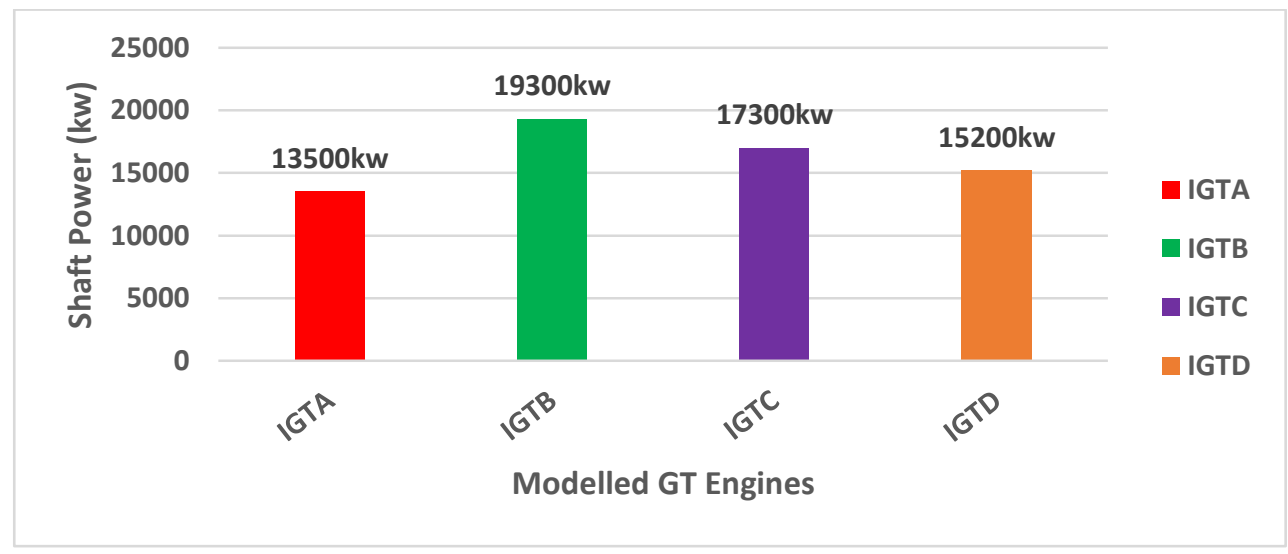

Fig 2: DP power of the modelled Engines

The selected GT engines were chosen based on the highest gas compressor power in all the three seasons of the year and in all the eighteen CS as analysed by Aziaka et al., [10] in Figure 3. The hot season shows the highest power requirement of the GC at each time of the day. It was based on this highest power that the prime mover (GT engine) was selected. The engine selection agrees with the work of Lubomirsky et al.,[11] as the gas compressor power in the hot season was chosen to be the basis of GT engine selection. 


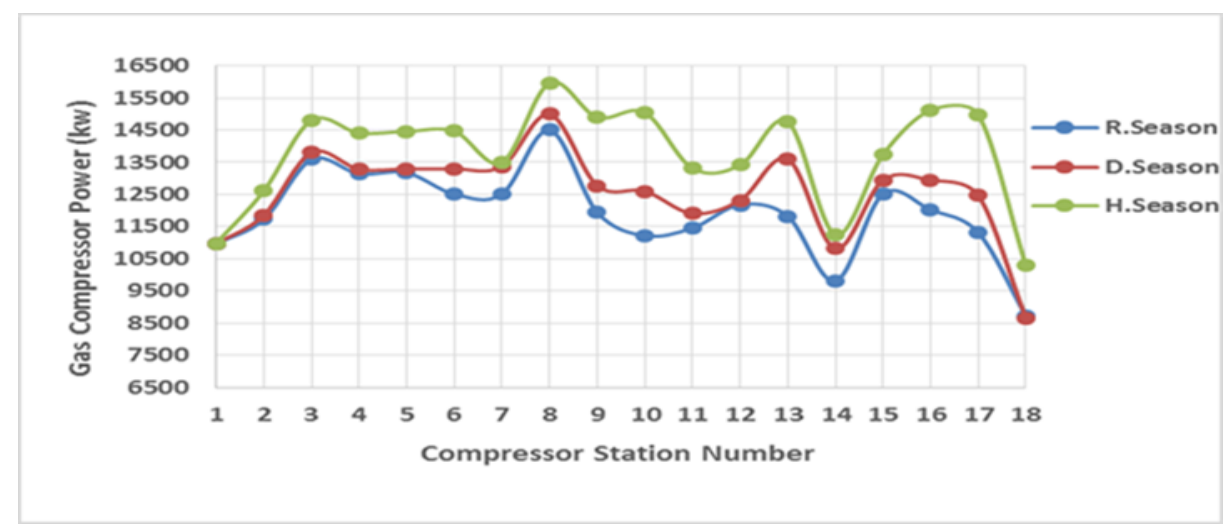

Fig 3: Gas compressor power for three seasons of the year [10]

\subsection{Map Scaling and Deteriorated Mode of the Engine}

Deterioration is introduced by reducing the scaling factors calculated in the design point. On the TURBOMATCH code, for $1 \%$ deterioration, a factor of 0.99 is been introduced, hence subsequent reduction in deteriorated value for a specific component follow the same trend.

$$
\begin{gathered}
E T A S F=\frac{\eta_{D P}}{\eta_{D P M a p}} \\
\mathrm{WASF}=\frac{W A C_{D P}}{W A C_{D P M a p}}
\end{gathered}
$$

\section{Deteriorated Compressor}

$$
\begin{aligned}
& \eta_{\text {Deteriorated }}=(1+\Delta \eta) * \mathrm{ETASF} * \eta_{\text {Map }} \\
& W_{\text {Deteriorated }}=(1+\Delta W A C) * W A S F * W A C_{\text {Map }}
\end{aligned}
$$

Where $\Delta \mathrm{n}$ stand for Deterioration of efficiency and $\triangle W A C$ - Deterioration of Mass flow function.

\section{For Clean Turbine}

The work function scaling factor is given by

$$
E H S F=\frac{D H_{D P}}{D H_{D P, M a p}} ; \quad D H_{N e w}=E H S F * D H_{M a p}
$$

The efficiency scaling factor is given by

$$
\text { ETASF }=\frac{\eta_{D P}}{\eta_{D P, \text { Map }}} ; \eta_{\text {New }}=E T A S F * \eta_{\text {MaP }}
$$

The flow function is given by

$$
T F S F=\frac{T F_{D P N e w}}{T F_{D P, M a p}} ; T F_{D P N e w}=T F S F * T F_{D P, \text { Map }}
$$




\section{For Deteriorated Turbine}

$$
\eta_{\text {Deteriorated }}=(1+\Delta \mathrm{\eta}) * E T A S F * \eta_{\text {Map }}
$$

Where,

$$
\begin{aligned}
& \Delta(D H) \text { - Deterioration of } \Delta H / T \\
& \Delta \eta-\text { Deterioration of efficiency } \\
& \Delta \mathrm{TF}-\text { Deterioration of mass flow function }
\end{aligned}
$$

\subsection{Conditions of Estimating the Number of the Gas turbine to be used for each Compressor Station}

The engines are expected to run on higher TET due to varying ambient temperature and increasing degradation at constant shaft power operation. The effect of higher TET as the engine continues to run on constant power was a concern in this research. For the engine to work efficiently and to accomplish the life of the project, increasing the number of the gas turbine in each station due to the impact of the ambient condition and degradation with the existing pipeline design became very imperative. The engine TET was fixed not to exceed $60 \mathrm{~K}$ above the DP value during operation. The $60 \mathrm{~K}$ TET was fixed such that irrespective of the adverse effect of engine degradation in addition with the harsh ambient temperature, at least one-eighth of the entire life of the engine can still be available for the engine based on the study of Gad-Briggs et al., [12]. However, most of the engines were modelled to run on path-load. The choice of fixing the $60 \mathrm{~K}$ above the DP was reasonable but was chosen arbitrarily. At baseline condition, Aziaka [7] investigated the influence of degradation on the number of gas turbine usage in natural gas pipeline network using the three degraded scenarios (optimistic, medium and pessimistic) including the clean condition at three years overhaul interval for the project life. He deduced that the clean, optimistic, medium and pessimistic scenarios used a total number of 90,99, 106 and 120, respectively of GT engine. Based on this investigation, the present research used two years overall interval to see how it will influence the number of GT to be used throughout the project life. 


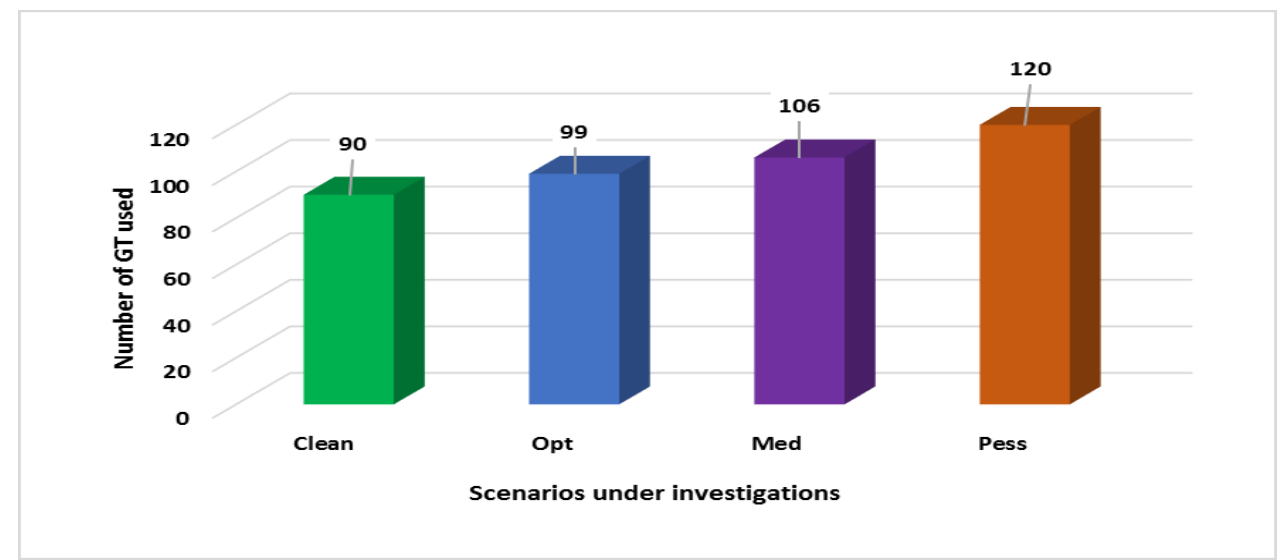

Fig 4: Total number of GT engines used at various scenarios for project life [7]

Equation (9) gives the developed equation that can evaluate the actual power of the GT engine and the corresponding number of the engine that can conveniently serve the GC power requirement for each CS throughout the project life. It should be noted in this equation that the required GT power for operation is inversely proportional to the number of GT per station. This implies that as the number of GT increases, the shaft power reduces and the summation of the reduced shaft power of the GT based on the actual number in each compressor station will serve the power requirement of the GC. Meanwhile, the original pipeline design remains the same even at the case of additional GT engine.

$$
G T_{p}=\frac{N G C * G C_{h p}}{N G T}
$$

Where,

$$
\begin{aligned}
G T_{p}= & \text { Required GT Power for operation } \\
N G C= & \text { Number of Gas Compressor per Station } \\
G C_{h p}= & \text { Highest Power of the GC per station based on season and time of day } \\
& \quad N G T=\text { Number of GT per station }
\end{aligned}
$$

Having ascertained the power and the number of GT engine to be used for each CS at clean condition, if the engine continues operation and at a certain year the engine TET increases above the limit due to the influence of elevated temperature and degradation, an additional engine will be added. A new power of all the engines will be achieved. This new power of the engine will be used to produce another engine model for a new simulation. The TET may still go higher after some time of operation due to the effect of elevated temperature and degradation until a convergence point where the simulated TET cannot be $60 \mathrm{~K}$ above the DP TET. This engine addition becomes necessary as it enhances continuous operation and serves as a palliative measure to improve the life and performance of the GT for the smooth 
operation of the engines and also to preserve the salvage value of the engines in the midst of harsh ambient temperature and degradation.

\subsection{Consideration of the Implanted Degradation base on Rescheduled-Overhaul (ROH)}

Three degraded scenarios were examined on the engines model, for the three scenarios in comparison with the clean condition. The three scenarios including the clean condition were investigated in each season (winter, dry and hot season) of the year with the implementation of a routine overhaul after two years interval for the project life of thirty (30) years different from the three years overhaul of the baseline condition.

In the developed engine model and for each season of each year throughout the project life, different values of degradation were implemented with consideration to the assumed two years routine overhaul. The percentage of values used was implanted with respect to industrial practice and literature as seen in gas turbine handbook [13], Naeem et.al., [14], Rainer et al.,[15], and Meher-Homji et.al.,[16]. All the scenarios were modelled for 30 years. Figure 5, Figure 6 and Figure 7 showed the percentage values of the implanted degradation for each scenario for winter, dry and hot seasons, respectively throughout the project life. In the winter season, as shown in Figure 5, opt ranges from $0.25 \%$ to $2.4 \%$, med ranges from $1.25 \%$ to $3.4 \%$ and pess ranges from $2.25 \%$ to $4.4 \%$. In the dry season, the implanted degradation for opt ranges from $0.5 \%$ to $2.65 \%$, med ranges from $1.5 \%$ to $3.65 \%$ while pessimistic ranges from $2.5 \%$ to $4.65 \%$. Finally, in the hot season, optimistic ranges from $0.75 \%$ to $2.9 \%$, the medium was between $1.75 \%$ and $3.9 \%$ while pessimistic ranges from $2.75 \%$ to $4.9 \%$. An interval of $0.75 \%$ between each year of the first two years of operation was assumed for all the seasons. Also, an interval of $1 \%$ between each scenario per season was assumed to account for the different level of degradation. These are shown in Figure 5 to Figure 7.

The years of the overhaul are year $3,5,7,9,11,13,15,17,19,21,23,25,27$, and 29. These years were chosen based on the assumption that the engine overhaul was implanted after two years interval for the project life. To account for the successive unrecovered degradation after each routine overhaul, the percentage from the specified range of values to be used depends on the scenario under investigation with their respective seasons. Therefore, $0.025 \%$ to $0.435 \%$ was implanted for the winter season to account for the years of the overhaul. To account for the unrecovered degradation after each overhaul for the opt scenario, $0.01 \%$ 
interval between each overhaul was adopted while $0.03 \%$ was used as the interval for both medium and pessimistic scenario in the winter season.

A similar approach was adopted for the dry and hot season with alternating values. The main aim was to show that after each maintenance cycle, the engine goes into operation with some percentage of unrecovered degradation. These values were used to reduce the flow capacity and isentropic efficiency of both the compressor, compressor turbine and power turbine of the engine.

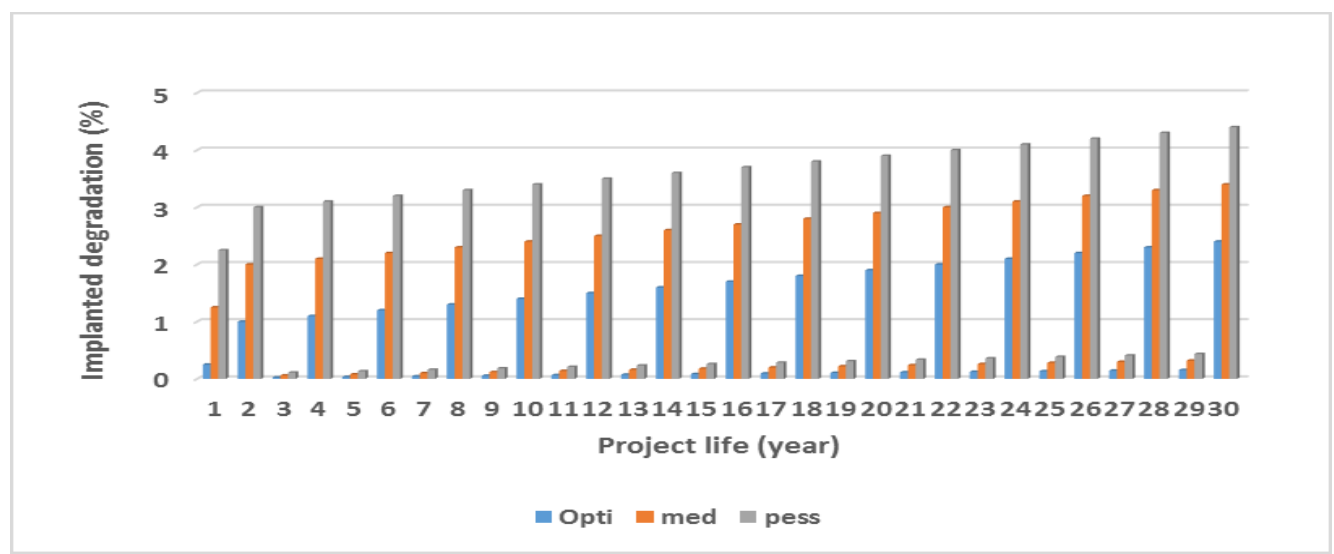

Fig 5 Implanted level of degradation for the winter season

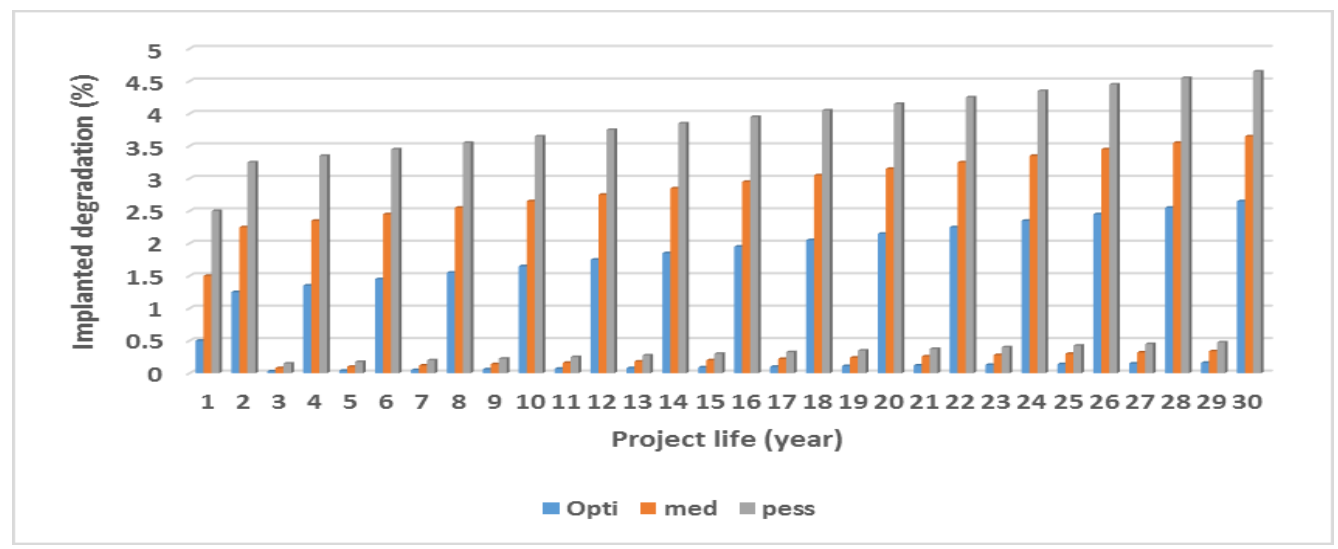

Fig 6: Implanted level of degradation for dry season 


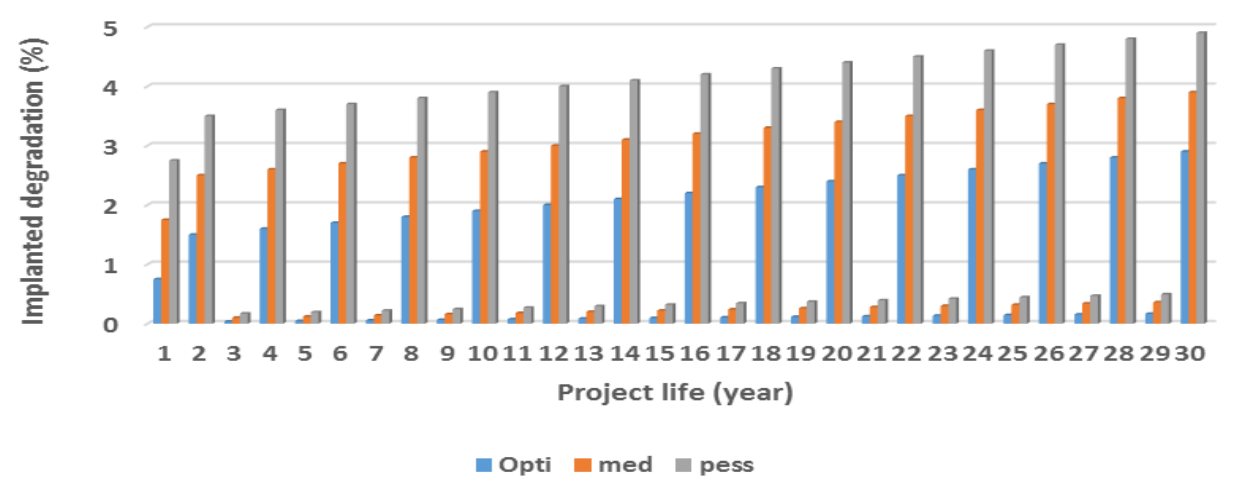

Fig 7: Implanted level of degradation for the hot season

\subsection{Result and Discussion}

Based on the operational limit set on the TET of the engines, the TET profile has been analysed in addition to other performance parameters in all the 18 compressor stations (CS). For instance, Figure 8 and Figure 9 showed the time-based TET profile map for CS 2 in the rainy season for the rescheduled-overhaul in comparison with the baseline condition. The result showed a reduction in TET for the $\mathrm{ROH}$ when compared with the baseline condition. The same trend was achieved in all the CS and all seasons with respect to the various scenarios under investigation. The baseline and ROH result for the TET profile showed the influence of degradation on engine operation at constant shaft power. So the higher the degradation, the higher the TET, vice Versa. The analysis will determine if an additional engine is needed for other scenarios to reduce the TET or not based on the engine condition of operation. The engine below was expected not to exceed TET of $1610 \mathrm{~K}$ since the DP was $1550 \mathrm{~K}$

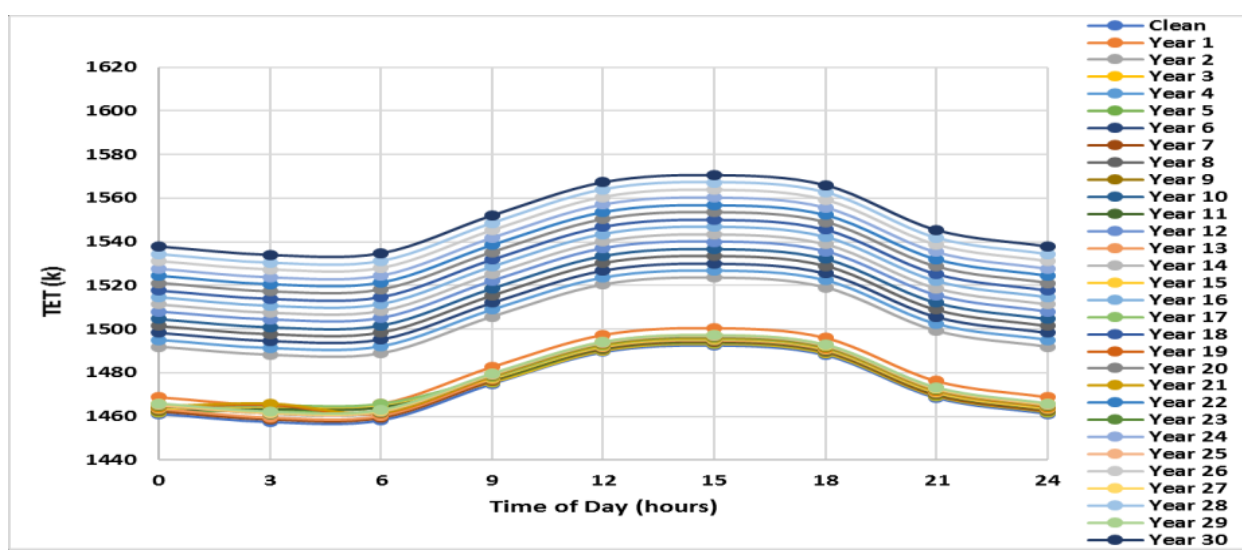

Fig 8: Time-based TET profile after ROH- Rainy season 


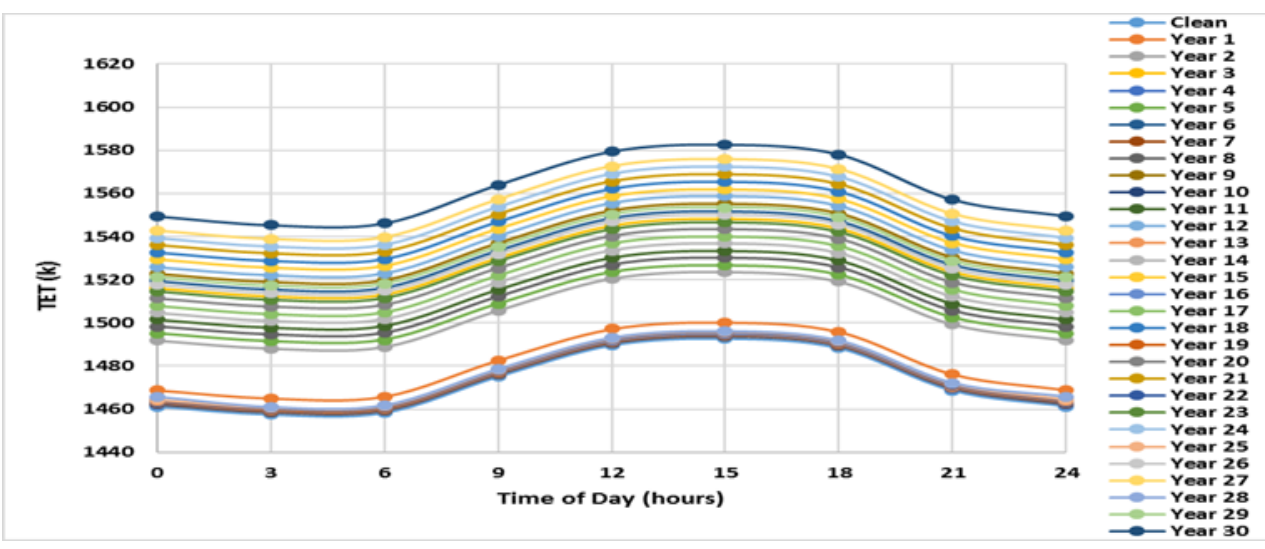

Fig 9: Time-based opt TET profile for the baseline condition -Rainy season

Figure 10 showed the influence of the re-scheduled overhaul in determining the number of engines usage in each year. The result indicated that the re-scheduled overhaul reduces the number of GT used when compared with the baseline. This is evidently shown in Figure 11, where only the pessimistic scenario is represented. The reason was that the ROH technique reduces the level of degradation of the engine. This means that at the 3rd year where degradation would have been on the increase, engine overhaul took place to hinder a further increase in degradation.

With this technique, an additional engine that would have come in as a result of an increase in degradation was hindered by the introduction of the overhaul. This technique helped to reduce the number of engine usage in each of the compressor stations. Figure 12 showed the reduced number of GT used in each of the CS. For instance, at baseline condition, CS 13 used 7 and 8 GTs for the medium and pessimistic scenario respectively, but at the implementation of $\mathrm{ROH}$, the number of GT reduced to 6 and 7 GT engines at the same CS 13 for the same scenario. The reduction of GT was also experienced by other CS. Meanwhile, the clean engine maintains the same number of engines throughout the project life in a similar way with the baseline case. This is because there was no degradation and overhaul for the clean engine operation.

It is necessary to state also that the outcome of the total number of GT used in each year for each of the CS was based on the power settings of the engines that will drive the GC. The operational TET of the engines was within the set limit. 


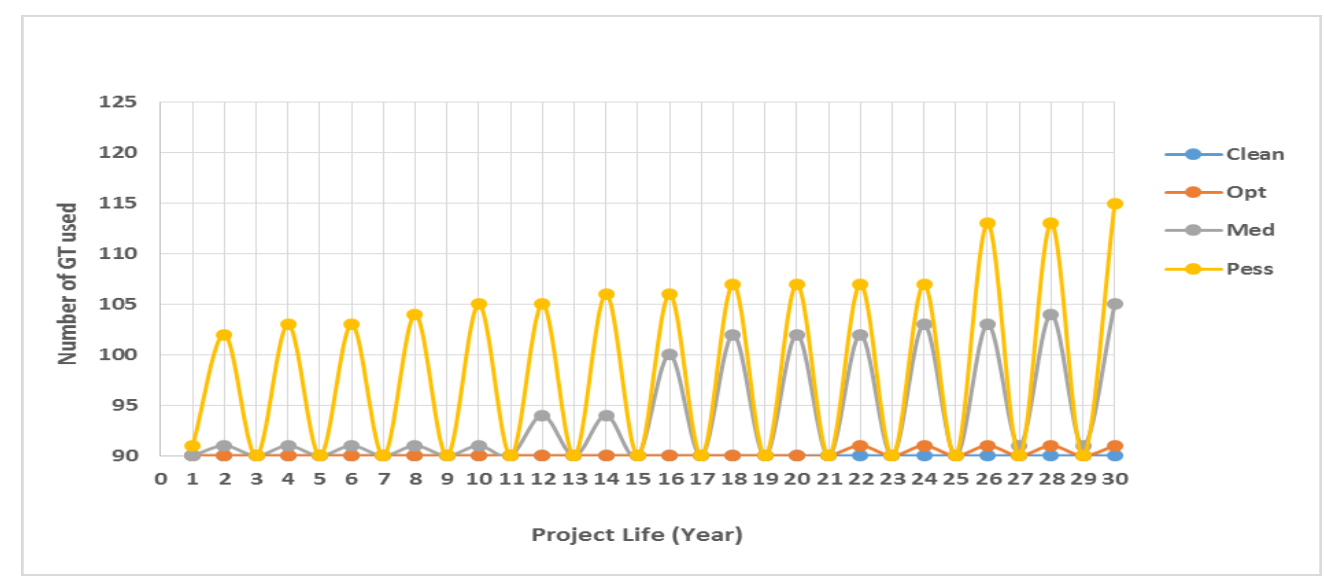

Fig 10: GT investment for the re-schedule overhaul

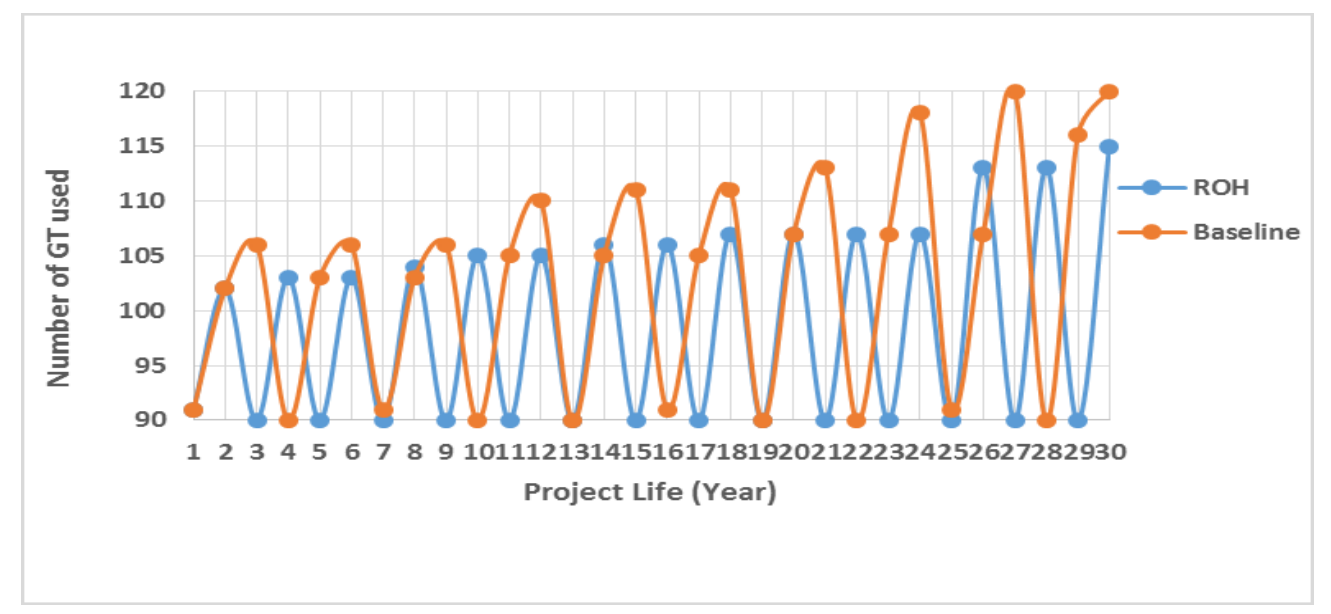

Fig 11: Number of engines used in the pessimistic scenario

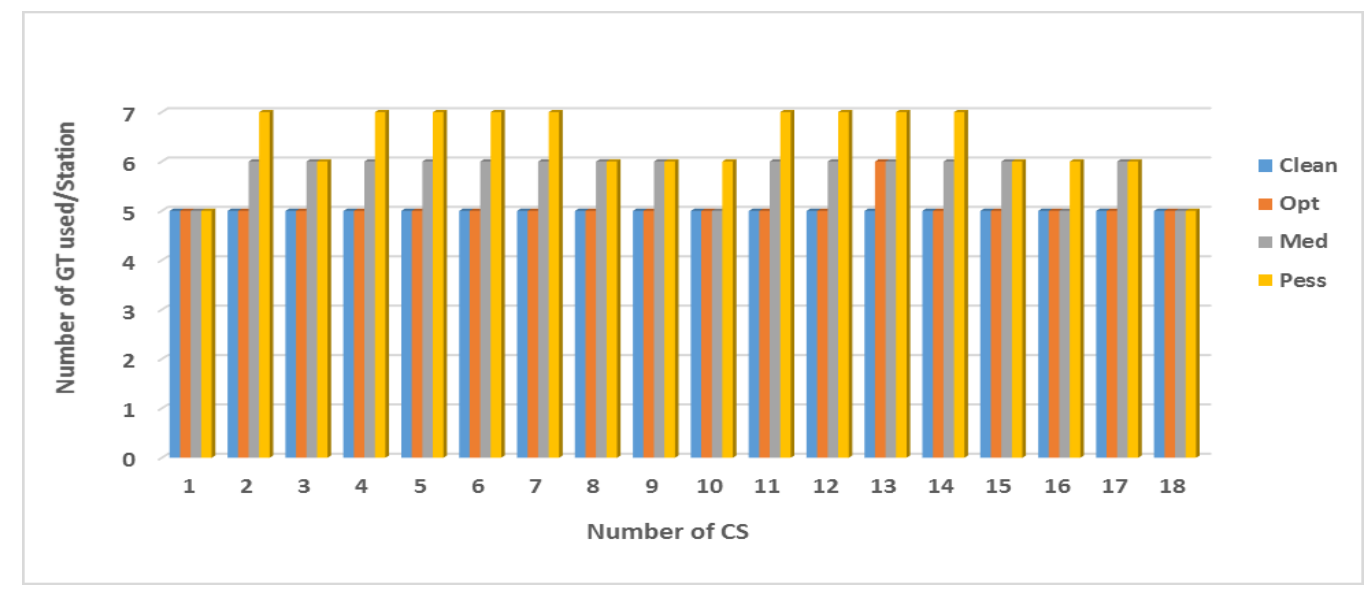

Fig 12: Number of GT used for each CS at different scenarios

Having evaluated the required number of GT in each CS, Figure 13 showed the total number of GT used in each scenario in comparison with the baseline case. The result showed that, the opt, med and pess scenario that used 99, 106 and 120 number of engines throughout the life 
of the engines for 18 compression stations at baseline reduces to 91, 104 and 115 respectively for the same number of compressor station and at the same operating conditions to deliver the same amount of gas as in the baseline case. The opt scenario had the highest reduction in the number of GT because the opt has a lower level of degradation. Having a lower level of degradation enabled the system to quickly get rid of the degraded condition at the introduction of the overhaul after two years of operation when compared with the waiting time of 3 years before overhaul. The number of engines was also reduced in other scenarios based on this phenomenon. Figure 14 showed the number of different engines used in the mixed fleets.

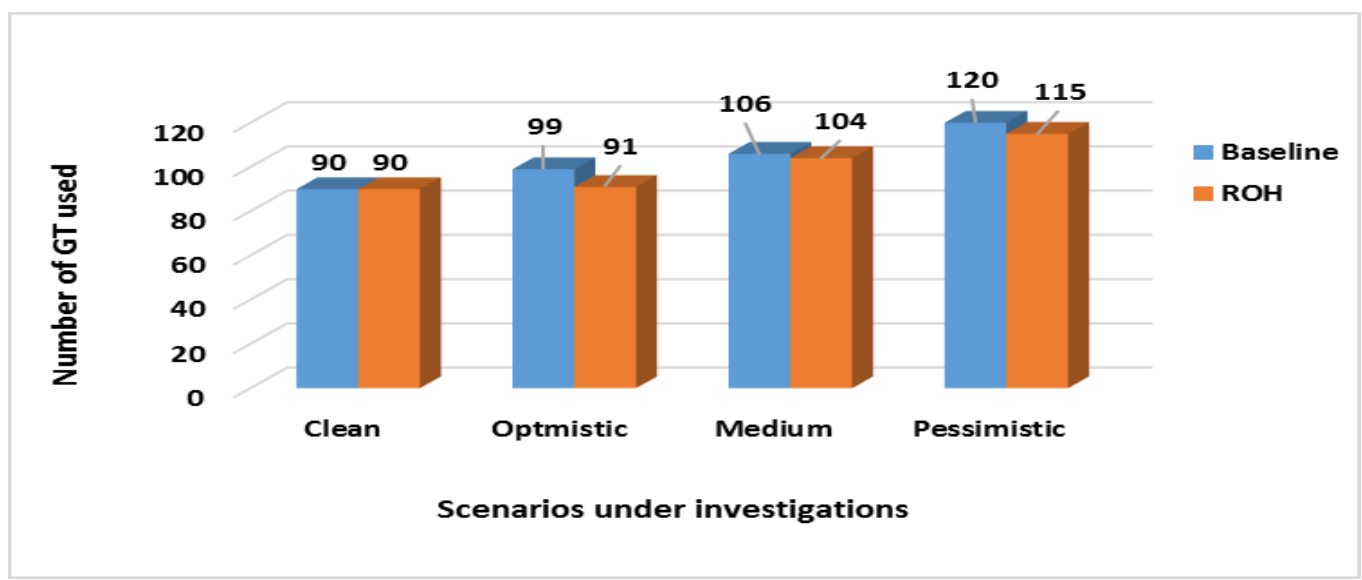

Fig 13: 1 GT used for ROH in comparison with the baseline

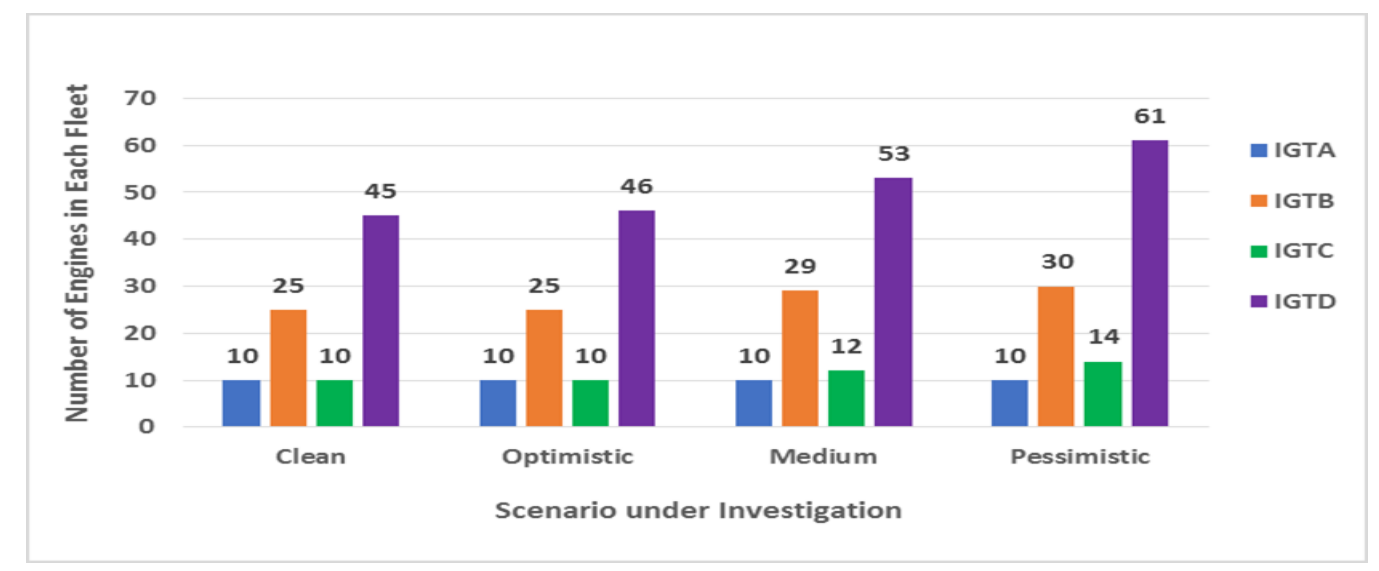

Fig 14: Number of each GT used in the mixed fleets for ROH 


\subsection{Conclusion}

The model to investigate the impacts of GT re-scheduled overhaul to determine the number of the gas turbine to be used in each compression station taking into account, time-based ambient condition, degradation and overhaul have been successfully developed and presented. The simulation of the developed engine model showed a very good correlation with actual engine data from the OEM with $99 \%$ accuracy thus indicating the suitability of the engine model performance prediction. TET profile maps of the various power settings of the engines that satisfied the condition of operation were produced. The produced TET map formed the bases of determining the exact number of GT to be used for project life. The result also showed a reduction in TET for the ROH when compared with the baseline condition. The result of the investigation also revealed that the implementation of two years overhaul interval enabled the engine not to undergo much degradation as in the case of three years interval thereby serving as life extension technique for gas turbine engine operating in fouled condition.

Significantly, the obtained result revealed that the opt, med and pess scenarios that used 99, 106 and 120 number of GT engines at baseline condition for the 18 CS reduced to 91, 104 and 115, respectively when $\mathrm{ROH}$ was implemented for the same number of CS and at the same operating conditions without altering the initial pipeline design.

The proposed approach will enhance engine life-extension strategies that engine life-cycle managers or natural gas pipeline investors may adopt to cost-effectively manage their engines while ensuring reliability and safety on the pipeline business. 


\subsection{Reference}

1. Aziaka D. S., Tukur N., Pilidis P. Techno-Economic Evaluation of Pipeline Compression System : Evaluation Of Gas Compressor Power In a Natural Gas Pipeline Network - Part 1. International Journal of Mechanical Engineering and Technology (IJMET). 2019; 10(11): 102-118.

2. Tukur N. Techno-Economics of Natural Gas Pipeline Compression System (2018). PhD Thesis. Cranfield University, Cranfield.United Kingdom; 2018.

3. Nasir A. Techno-Economic Study of Gas Turbine in Pipeline Applications. Cranfield University; 2013.

4. Beal L. The Routine Overhaul of Natural Gas-Fired Combustion Turbines in Interstate Natural Gas Transmission Service. 2004; (December). Available at: file:///Z:/Overhaul paper.pdf

5. Kurz R., Brun K. Gas turbine tutorial-Maintenance and operating practices effects on degradation and life. Proceedings of 36th Turbomachinery. 2007; : 173-185.

6. NSPS proposal. Turbine Replacement due to Routine Maintenance: NSPS Proposal Support Document, EPA-450/2-77-017a. 1977.

7. Aziaka D. S. Techno-Economic Study for Degraded gas turbine on pipeline application in the Oil and Gas Industry. Cranfield University, Bedfordshire, United Kingdom; 2019.

8. Wulf RH. Engine Diagnostics Program: CF6-50 Engine Performance DeteriorationNASA CR-159867, conducted by the General Electric Company, Aircraft Engine Business Group. 1980.

9. GE Engines. Gas turbine for Land and Marine application. 2016. Available at: https://pdf.nauticexpo.com/pdf/ge-marine/lm-1600/31041-11949.html (Accessed: 20 November 2016)

10. Aziaka D. S., Tukur N., Pilidis P. Techno-Economic Evaluation of Pipeline Compression System : Gas Compressor Operation Evaluation on Natural Gas Pipeline Network-Part 2. International Journal of Mechanical Engineering and Technology (IJMET). 2019; 10(11): 119-132. Available at: http://iaeme.com/MasterAdmin/UploadFolder/IJMET_10_11_011/IJMET_10_11_011. pdf

11. Lubomirsky, M., Kurz, R., Klimov, P. and Mokhatab S. Configuration impact availability, fuel consumption and pipeline capacity. Pipeline and Gas Journal. 2010; 237(1): 48-54. 
12. A. Gad-Briggs, A. Haslam PL. Effect of change in the role of an aircraft on engine life. The Aeronautical Journal. 2013; 117(1196): 1053-1070.

13. Meherwan; P.Boyce. Gas turbine Engineering Handbook. Third Edit. Gulf Professional Publishing, 30 Corporate Drive, Suite 400, Burlington, MA 01803, USA; 2006. 101-103 p.

14. Naeem M., Singh R., Probert D. Impacts of aero-engine deteriorations on military aircraft mission's effectiveness. Aeronautical Journal. 2001; 105(1054): 685-695.

15. Kurz R., Brun K. Degradation Effects on Industrial Gas Turbines. 2008;

16. Onari MM. 43 rd Turbomachinery \& 30 th Pump Users Symposia ( Pump \& Turbo 2014 ) September 23-25, 2014 | Houston, TX | pump turbo . tamu . edu. 2014; (1998). 